\title{
DESEMPEÑO LABORATORIAL DE LAS UNIDADES DE ATENCIÓN PRIMARIA EN EL DIAGNÓSTICO DE TUBERCULOSIS EN PELOTAS, BRASIL
}

\author{
Dagoberta Alves Vieira Beduhn ${ }^{1, a}$, Jenifer Harter ${ }^{1, a}$, Simone Pieren dos Reis ${ }^{1, b}$, Luize Barbosa Antunes ${ }^{2, c}$, \\ Roxana Isabel Cardozo-Gonzales ${ }^{2, d}$
}

\begin{abstract}
RESUMEN
Con el objetivo de evaluar el desempeño laboratorial de las unidades de atención primaria (UAP) en el diagnóstico de tuberculosis por baciloscopía en Pelotas, Brasil, se realizó un estudio descriptivo en el cual se incluyó todas las baciloscopías solicitadas en once UAP entre 2007 y 2010. Para la evaluación del desempeño laboratorial se cuantificó el número de baciloscopías solicitadas y el tiempo entre la solicitud y la lectura de la lámina de examen en el laboratorio de referencia. Se encontraron 477 solicitudes registradas en las UAP. De ese total, apenas $51,8 \%$ fueron encontradas en el libro de registro del laboratorio, el desempeño más bajo fue de $24,2 \%$ y el más alto de $71,4 \%$. Se identificó, en relación al tiempo de lectura de las láminas, que el $27,7 \%$ de las lecturas demoraban 8 días o más. Se concluye que las UAP evaluadas de la ciudad de Pelotas tienen un bajo desempeño laboratorial en el diagnóstico de TB por baciloscopías.
\end{abstract}

Palabras clave: Tuberculosis; Laboratorios; Evaluación de proceso (atención de salud); Atención primaria de salud (fuente: DeCS BIREME).

\section{LABORATORY PERFORMANCE OF PRIMARY CARE UNITS IN TUBERCULOSIS DIAGNOSIS IN PELOTAS, BRAZIL}

\begin{abstract}
In order to assess laboratory performance of Primary Care Units (UAP, Spanish acronym) in TB diagnosis though the sputum smear test in Pelotas, Brazil, a descriptive study was completed which included all sputum smear tests requested at eleven primary care units from 2007 to 2010 . To assess laboratory performance, the number of sputum smear tests requested was quantified as well as the time between the request and the reading of the glass slides at the lab. 477 requests were registered at UAP. Of this total, only $51,8 \%$ were found in the laboratory's record book, the lowest performance was $24,2 \%$ and the highest $71,4 \%$. Regarding the reading of glass slides, it was determined that $27,7 \%$ readings took 8 days or more. It is concluded that the primary care units in Pelotas have a low laboratory performance in TB diagnosis by sputum smear tests.
\end{abstract}

Key words: Tuberculosis; Laboratories; Process assessment (health care); Primary health care (source: MeSH NLM).

\section{INTRODUCCIÓN}

La tuberculosis (TB) continúa siendo un grave problema de salud pública mundial, a pesar de ser una enfermedad infecciosa prevenible y curable ${ }^{(1)}$. Se estima que un tercio de la población está infectada por el Mycobacterium tuberculosis, bacilo responsable de la TB. Se ha descrito, además, que al menos el 10\% de los infectados desarrollan la enfermedad, y es la tos persistente el síntoma principal, la cual puede ser seca o productiva ${ }^{(2-4)}$. El diagnóstico precoz y el tratamiento inmediato son las principales medidas de control. El examen de diagnóstico más utilizado es la baciloscopía de esputo. Un método simple y seguro que, ejecutado correctamente en todas sus fases, detecta de 60 a $80 \%$ de los casos de TB pulmonar; la cual, por ser bacilífera, en la mayoría de los casos representa la principal fuente de infección y mantenimiento de la cadena de transmisión (2).

En Brasil, el proceso de descentralización de las acciones y servicios para los sistemas locales de salud, contempló la ampliación de las acciones de detección de casos de TB en las unidades de atención primaria (UAP) ${ }^{(5)}$. La detección

\footnotetext{
Programa de Pós-Graduação em Enfermagem, Universidade Federal de Pelotas. Pelotas, Brasil.

Universidade Federal de Pelotas. Pelotas, Brasil.

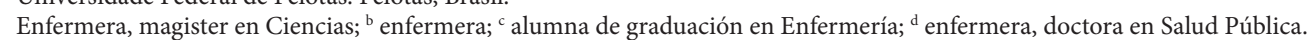

Recibido: 10-05-2013 Aprobado: 04-09-13
}

Citar como: Beduhn DAV, Harter J, dos Reis SP, Barbosa Antunes L, Cardozo-Gonzales RI. Desempeño laboratorial de las unidades de atención primaria en el diagnóstico de tuberculosis en Pelotas, Brasil. Rev Peru Med Exp Salud Publica. 2013;30(4):621-5. 
precoz de casos de TB en las UAP es fundamental para la identificación de las fuentes de infección, tratamiento e interrupción de la cadena de transmisión ${ }^{(2,6)}$. Esa acción, busca identificar al sintomático respiratorio (SR) en los servicios de salud o en la comunidad ${ }^{(2)}$. La identificación del SR de forma activa (busca en la comunidad) o pasiva (demanda espontanea de las personas) puede ser realizada por cualquier profesional de salud capacitado conforme recomendación del Programa Nacional de Control de la TB. Cuando el establecimiento de salud cuenta con la estrategia "salud de la familia" o tiene el "programa de promotores de salud" la captación de SR es realizada principalmente por promotores de salud. En ambos casos, los profesionales necesitan recibir capacitación, ser receptivos y atentos para observar los signos de la enfermedad detectarlos precozmente reduciendo el riesgo de transmisión ${ }^{(2,7)}$.

El Municipio de Pelotas es el tercero más poblado del estado de Rio Grande do Sul (Brasil) con 339934 habitantes ${ }^{(8)}$. Tiene 51 UAP, un Programa Municipal de Control de la TB y un Laboratorio Municipal (referencia para realización de la baciloscopía de esputo). La ciudad de Pelotas es prioritaria para el desarrollo de acciones que promuevan el control de la TB. En el periodo comprendido entre 2006 y 2010, presentó un número promedio de casos (45,6 casos por 100 mil hab.) superior a la tasa nacional (38,2 casos por 100 mil hab.). Dada la relevancia de la detección de casos de TB en el control de la enfermedad, el presente estudio tuvo como objetivo analizar el desempeño laboratorial de los servicios de APS, en la evaluación del SR y la agilidad en el diagnóstico de la enfermedad por medio del examen de baciloscopía, en la cuidad de Pelotas, Brasil.

\section{EL ESTUDIO}

Se realizó un estudio transversal en la ciudad de Pelotas, de enero de 2007 a diciembre de 2010, en el cual participaron once UAP, localizadas en los barrios que presentaron mayor prevalencia de casos de TB de acuerdo con datos de la vigilancia epidemiológica ${ }^{(9)}$ y el laboratorio municipal de referencia (LMR). Las UAP estaban distribuidas en los cuatro distritos sanitarios urbanos existentes en la ciudad según se detalla a continuación: Fragata (4); Tres Vendas (3); Centro/ Várzea (3), y Areal/Praias (1).

La información sobre las solicitudes de baciloscopías se recuperó de los libros de registro de SR de las UAP y aquella información en cuanto a las solicitudes recibidas fue recogida del libro de registro del LMR. Se verificó la recepción de las solicitudes de baciloscopías por el LMR, cruzando los datos de exámenes solicitados y realizados por el laboratorio. El indicador de desempeño laboratorial durante la identificación de SR en los servicios seleccionados se calculó empleando la siguiente formula: (número de baciloscopías recibidas en el LMR / número de baciloscopías solicitadas por las UAP) x 100. Además, se calculó el tiempo entre la solicitud de la baciloscopía y la lectura de la lámina se analizó como indicador de agilidad del diagnóstico. Cabe señalar que el desempeño del LMR no fue objeto de evaluación en esta investigación.

Para el análisis estadístico se calculó las frecuencias del número de solicitudes de baciloscopía por UAP, el número de baciloscopías recibidas y examinadas por el laboratorio distribuidas por UAP, y del tiempo (días) entre la fecha de solicitud del examen y la fecha del resultado (fecha de lectura de la lámina) en el laboratorio. El estudio contó con la aprobación del Comité de Ética de la Facultad de Enfermería de la Universidad Federal de Pelotas y de la Secretaría de Salud de Pelotas. Durante el desarrollo del trabajo se cumplió con los principios éticos de la investigación en salud. Para guardar la confidencialidad de las UAP evaluadas, la información fue codificada de A a G por orden decreciente del número de solicitudes de baciloscopías.

\section{HALLAZGOS}

De las once UAP seleccionadas, dos carecían del libro de SR, una no presentaba registros en su libro y una no tenía datos referentes al periodo de estudio. En las siete UAP restantes se identificaron 477 baciloscopías solicitadas. De ellas, el 51,8\% (247/477) fueron encontradas en el libro de registro del LMR. En ninguna de las UAP evaluadas se encontró un desempeño del $100 \%$, se encontró que el desempeño más bajo fue de $24,2 \%$ y el más alto de $71,4 \%$ (Tabla 1 ).

Tabla 1. Desempeño laboratorial de las unidades de atención primaria en la evaluación laboratorial del sintomático respiratorio en Pelotas (Brasil), 2007-2010

\begin{tabular}{cccc}
\hline Unidad & $\begin{array}{c}\text { Baciloscopías } \\
\text { solicitadas } \\
\mathbf{n = 4 7 7}(\%)\end{array}$ & $\begin{array}{c}\text { Baciloscopías } \\
\text { recibidas } \\
\mathbf{n = 2 7 7}(\%)\end{array}$ & $\begin{array}{c}\text { Indicador de } \\
\text { desempeño* } \\
\text { (\%) }\end{array}$ \\
\hline A & $110(23,1)$ & $56(11,7)$ & 50,9 \\
B & $95(19,9)$ & $62(13)$ & 65,3 \\
C & $91(19,1)$ & $22(4,6)$ & 24,2 \\
D & $81(17,0)$ & $42(8,8)$ & 51,9 \\
E & $44(9,2)$ & $28(5,9)$ & 63,6 \\
F & $35(7,3)$ & $25(5,2)$ & 71,4 \\
G & $21(4,4)$ & $12(2,5)$ & 57,1 \\
\hline
\end{tabular}

* Para el cálculo del indicador desempeño se empleó la siguiente formula: (número de baciloscopías recibidas en el LMR / número de baciloscopias solicitadas por las UAP) $\times 100$ 
Para la evaluación de la agilidad en el diagnóstico de TB por baciloscopías se consideraron solo 209 solicitudes de baciloscopía del total de 477, debido a la falta de la fecha en los registros de las UAP. Se encontró que el $48,8 \%(102 / 209)$ de las lecturas fueron realizados en un tiempo de 0 a 3 días; el 23,4\% (49/209), de 4 a 7 días; y el $27,8 \%$ (58/209), en un tiempo mayor o igual a 8 días.

\section{DISCUSIÓN}

La calidad de la información en salud permite subsidiar decisiones clínicas, administrativas y de apoyo a la investigación, se contribuye asi a la organización de los servicios y al enfrentamiento a las enfermedades ${ }^{(10)}$. Los problemas en el proceso de producción de la información referente a la TB, como la falta de datos de las acciones de salud realizadas, evidencian problemas de gestión, lo que puede comprometer el planeamiento y desarrollo de la búsqueda de SR y del diagnóstico precoz ${ }^{(11)}$.

De acuerdo con las recomendaciones del Programa Nacional de Control de TB (PNCT) los libros de registro de SR deben estar presentes en todas las UAP, deben ser llenados correctamente y actualizados regularmente (2). Los resultados del presente estudio revelan problemas tanto en el llenado de este libro, e incluso ausencia de él. Resultados semejantes fueron identificados en un estudio realizado en otros dos municipios brasileños ${ }^{(12)}$. Tal fragilidad tiene repercusión en la evaluación, planeamiento y ejecución de las acciones de control e inclusive favorece la subnotificación de SR evaluados en el municipio.

Un estudio realizado con casos nuevos de TB en una UAP de Rio de Janeiro identificó que el $14,1 \%$ de las solicitudes de baciloscopías no eran realizadas ${ }^{(13)}$, lo cual representa un menor porcentaje a lo encontrado en este estudio. Si consideramos que se espera encontrar un 4\% de individuos con baciloscopía positiva entre los SR ${ }^{(2)}$, es probable que entre las personas a las que no le realizaron el examen, aun cuando hubiesen dejado una muestra de esputo, haya algún caso positivo. Esto constituye una importante fuente de infección, a ello se debe sumar que cada persona infectada sin tratamiento puede contaminar en promedio de 10 a 15 personas por año ${ }^{(14)}$. De allí la importancia de evaluar y mejorar el desempeño en el diagnóstico de TB por parte de las UAP.

La pérdida considerable de información en la recepción de la baciloscopía por el laboratorio puede deberse a la responsabilidad dada al SR de entregar las muestras al LMR, hecho que va contra la normativa del PNCT. Una investigación realizada en 38 municipios del estado de Goiás, mostró que solo el $7,9 \%$ del flujo de muestras de esputo fue adecuado. El principal problema encontrado estaba relacionado con la responsabilidad atribuida al usuario de entregar la muestra de esputo al laboratorio y de recoger los resultados del examen (11). Se ha descrito, además, que cuando es necesario el desplazamiento del usuario, para la obtención del diagnóstico de TB, se genera una mayor demora en la obtención del resultado ${ }^{(15)}$.

La comunidad científica viene desplegando esfuerzos para encontrar pruebas diagnósticas más rápidas, económicas y altamente factibles de ser utilizadas en los servicios. De esta manera, sería posible la realización del examen en el momento de la sospecha de la enfermedad ${ }^{(16)}$, consecuentemente, reducirían el tiempo entre la identificación del SR y el diagnóstico. Sin embargo, mientras esta tecnología no llegue a las UAP, es prioridad dar facilidades a los SR para que realicen la baciloscopía inmediatamente después de la solicitud. La responsabilidad de recolecta, conservación y transporte del esputo es de la UAP solicitante. Por lo tanto, corresponde a la administración municipal establecer el flujo y subsidiar la realización de esta acción ${ }^{(2)}$. Los resultados de presente estudio evidenciaron que las UAP no garantizaron la realización de la baciloscopía para todos los SR conforme recomendación del PNCT ${ }^{(2)}$, revelando debilidades en su desempeño.

Por otro lado, se ha descrito también que la estructura de las UAP podría influenciar en el desempeño del diagnóstico de TB. Un estudio señaló que en la mayoría de las UAP no existe un local adecuado para recolecta y el acondicionamiento del esputo, y que solo el $44,5 \%$ de las establecimientos evaluados contaban con refrigeradora o caja térmica para el almacenamiento de muestras ${ }^{(17)}$. Otras deficiencias como el desabastecimiento de insumos (falta de envases para colecta de esputo), y la falta de un flujo preestablecido para transporte de las muestras de la unidad solicitante hasta el laboratorio, ya fueron descritos como causas de retardo en el diagnóstico de la enfermedad (18).

En lo referente a la agilidad en el diagnóstico, los tiempos más frecuentes fueron considerados prolongados, ya que el PNCT recomienda que después de la solicitud, el resultado del examen sea disponible entre 24 y 48 horas para el equipo de salud, para así iniciar inmediatamente el tratamiento para los casos positivos (2). Este tiempo amplio puede contribuir al retardo del inicio del tratamiento y a la propagación de la enfermedad entre los contactos, dificultando el control de la TB. De acuerdo con un estudio realizado en Nova Iguaçu, Brasil, el tiempo entre la entrada del paciente en el sistema de salud (primera atención en una unidad) y el inicio del tratamiento para TB fue considerado como excesivo, lo que sugiere la necesidad de estrategias para la reducción de este tiempo ${ }^{(19)}$. 
El transporte de muestras de esputo en la ciudad de Pelotas necesita ser replanteado y así garantizar la realización de la baciloscopía para el diagnóstico precoz e inicio del tratamiento. Es necesario desarrollar acciones de sensibilización a los profesionales de salud en cuanto la importancia de llenado y utilización de los registros. Se considera fundamental avanzar en la creación de un sistema de información basado en tecnologías de la información y comunicación disponibles en los servicios de salud con la finalidad de obtener facilidades para implementar estrategias más efectivas para la identificación del SR y la realización del examen de baciloscopía en tiempo oportuno en las UAP.

Las limitaciones del estudio son atribuidas a la utilización de fuentes secundarias, a la falta de registros de las fechas en los libros y a la selección de indicadores restrictos a algunos de los aspectos del desempeño de las UAP. A pesar de no evidenciar las causas que pueden haber determinado el bajo desempeño de los servicios, es posible identificar las debilidades en el alcance de las metas propuestas.
Se concluye que las UAP evaluadas de la ciudad de Pelotas tienen un bajo desempeño laboratorial en el diagnóstico de TB por baciloscopías, lo cual incrementa la posibilidad de la existencia de SR con TB no diagnosticado, y consiguiente incremento del riesgo de diseminación de la enfermedad por parte de estos. Se requiere evaluar los procesos de evaluación y manejo del SR, así como de la lectura de baciloscopías, para elaborar estrategias que permitan mejorar el diagnóstico de las personas con TB y permitan brindarles un tratamiento oportuno.

Contribuciones de autoría: DB, JH y RCG participaron en la concepción y diseño del artículo, en la recolección y obtención de resultados, en el análisis e interpretación de datos y en la redacción del artículo. JH y RCG participaron de la asesoría estadística, técnica y administrativa. SP contribuyó con la revisión crítica del artículo y LB participó de la recolección y obtención de resultados. Todos autores aprobaron la versión final del artículo.

Fuentes de financiamento: Conselho Nacional de Desenvolvimento Científico e Tecnológico (CNPq) - Brasil

Conflictos de interés: los autores declaran no tener conflictos de interés.

\section{REFERENCIAS BIBLIOGRÁFICAS}

1. World Health Organization. Global tuberculosis report 2012. Geneva: WHO; 2012.

2. Brasil, Ministério da Saúde, Secretaria de Vigilância em Saúde, Departamento de Vigilância Epidemiológica. Manual de recomendaçôes para o controle da tuberculose no Brasil. Brasília: Ministério da Saúde; 2011.

3. Campos HS. Etiopatogenia da tuberculose e formas clínicas. Pulmão RJ. 2006;15(1):29-35.

4. World Health Organization. Global tuberculosis control: epidemiology, strategy, financing [Internet]. Geneva: WHO; 2009 [citado el 28 de marzo de 2013]. Disponible en: http://www.who.int/tb/ publications/global_report/2009/ en/

5. Brasil, Ministério da Saúde, Secretaria de Atenção à Saúde, Departamento de Atenção Básica. Política Nacional de Atenção Básica. Brasília: Ministério da Saúde; 2006.

6. Chadambuka A, Mabaera B, Tshimanga M, Shambira G, Gombe
NT, Chimusoro A. Low tuberculosis case detection in Gokwe North and South, Zimbabwe in 2006. Afr Health Sci. 2011;11(2):190-6.

7. Marcolino ABL, Nogueira JA, Ruffino-Netto A, Moraes RM, Sá LD, Villa TCS, et al. Avaliação do acesso às açôes de controle da tuberculose no contexto das equipes de saúde da família de Bayeux-PB. Rev Bras Epidemiol. 2009;12(2):144-57.

8. Sala de apoio à Gestão [Internet]. Brasília: Ministério da Saúde; 2013 [citado el 20 de agosto de 2013]. Disponible en http://189.28.128.178/sage/

9. Secretaria Municipal de Saúde. Vigilância Epidemiológica. Distribuição dos casos de Tuberculose no Município de Pelotas de 2007 2010. Pelotas: Municipal de Saúde; 2012.

10. Donabedian A. The quality of care. How can it be assessed? JAMA. 1988;260(12):1743-8.

11. Moreira MAC, Bello AS, Melo CALPS, Silva MV, Lorusso V. Análise de aspectos relacionados ao desempenho do Programa de Controle da Tuberculose em municípios do Estado de Goiás. Bol Pneumol Sanit. 2006;14(3):153-8.

12. Scatolin BE. A busca de sintomático respiratórios de tuberculose pelo Agente Comunitário de Saúde em dois municípios prioritários: Natal e Ribeirão Preto. [Dissertação]. São Paulo: Universidade de São Paulo, 2012.

13. Filha MMT, Daumas RP, Alves LC, Leimann BCQ, Engstrom EM. Análise da tuberculose em uma unidade de Atenção Primária à Saúde na cidade do Rio de Janeiro: perfil clínico, resultado de tratamento e qualidade dos registros. Cad Saúde Colet. 2012;20(2):169-76.

14. Brasil, Ministério da Saúde, Secretaria de Vigilância em Saúde. Guia de Vigilância epidemiológica. 6a edição. Brasília: Ministério da Saúde, 2005.

15. Gele AA, Sagbakken M, Abebe F, Bjune GA. Barriers to tuberculosis care: a qualitative study among Somali pastoralists in Ethiopia. BMC Res Notes. 2010;3:86. 
16. Machado ACFT, Steffen RE, Oxlade O, Menzies D, Kritski A, Trajman A. Fatores associados ao atraso no diagnóstico da tuberculose pulmonar no estado do Rio de Janeiro. J Bras Pneumol. 2011;37(4):512-20.

17. Dantas DNA, Silva MPM, Oliveira DRC, Enders BC, Paiva REA, Arcêncio RA. Ações do agente comunitário de saúde no diagnóstico da tuberculose pulmonar. Rev Rene. 2011;12:980-7.
18. Nogueira JA, Ruffino Netto A, Monroe AA, Gonzales RIC, Villa TCS. Busca ativa de sintomáticos respiratórios no controle da tuberculose na percepção do Agente Comunitário de Saúde. Revista Eletrônica de Enfermagem [Internet]. 2007[citado el 28 de enero de 2013];9(1):106-118. Disponible en: http://www.fen.ufg.br/revista/v9/ n1/pdf/v9n1a08.pdf

19. Maior ML. Tempo entre o início dos sintomas e o tratamento de tuberculose pulmonar em um município com elevada incidência da doença. J Bras Pneumol. 2012;38(2):202-9.

Correspondencia: Dagoberta Alves Viera Bedubn.

Dirección: Rua Visconde de Sinimbú, 526Vila Castilho, CEP 96020-790- PelotasRio Grande do Sul /Brasil. Teléfono: 53-91138043

Correo electrónico: daguialvesvieira@ hotmail.com

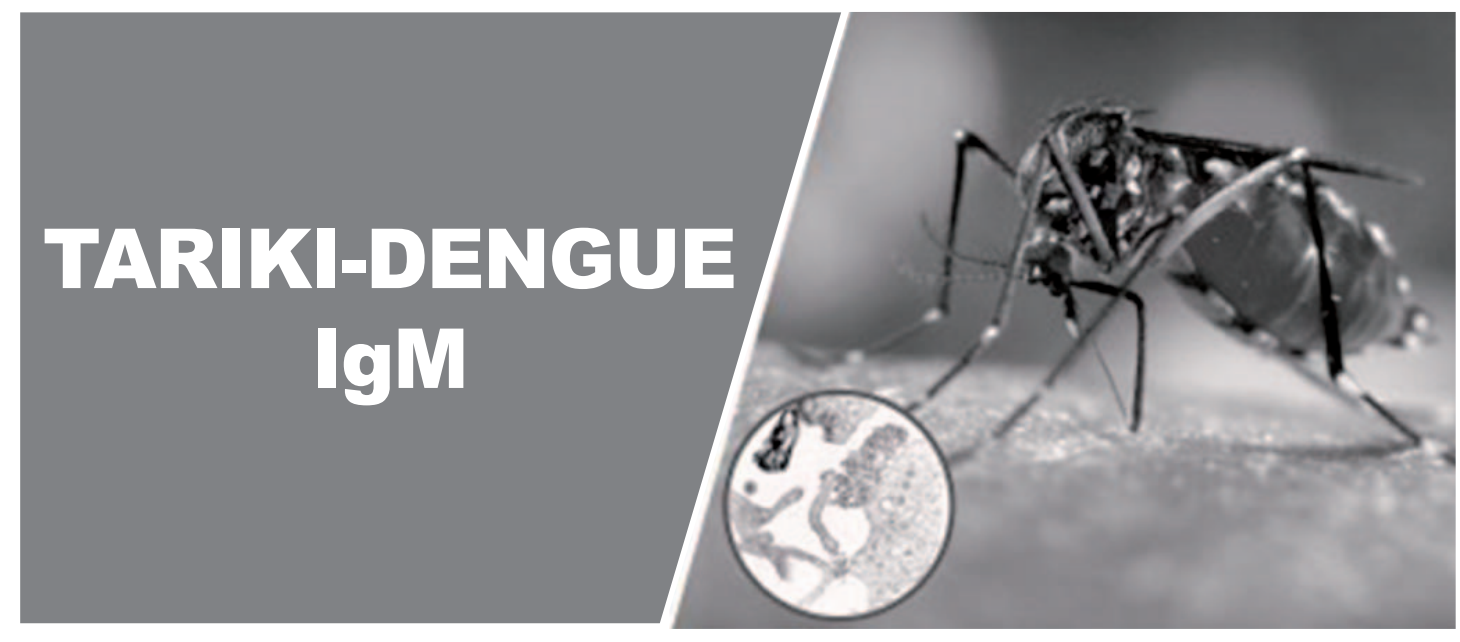

\section{ELISA de CAPTURA IgM DENGUE}

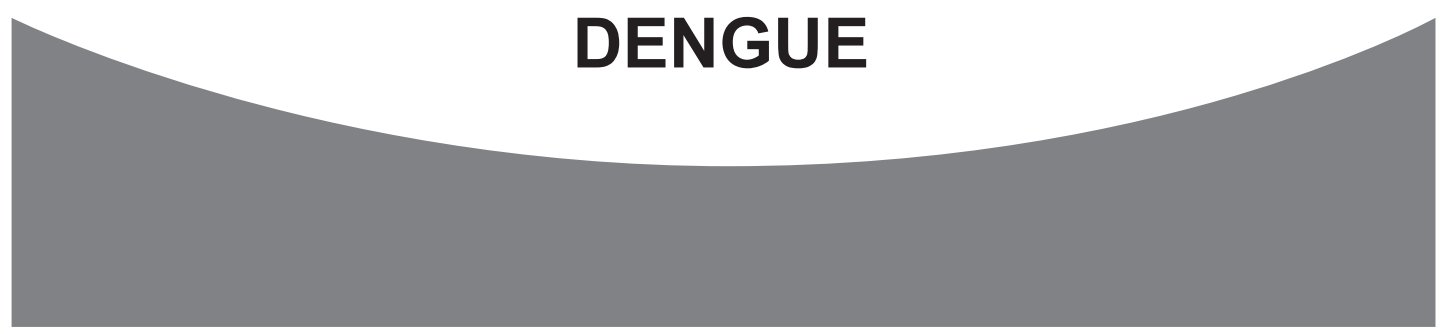

\section{TARIKI: disponible en el Perú}

Kit para la determinación de anticuerpos IgM anti Dengue, desarrollado y producido por el Instituto Nacional de Salud, Perú INS/MINSA 